\title{
An Alternative Estimator for Estimating the Finite Population Mean Using Auxiliary Information in Sample Surveys
}

\author{
Ramkrishna S. Solanki, Housila P. Singh, and Anjana Rathour \\ School of Studies in Statistics, Vikram University, Ujjain 456010, India \\ Correspondence should be addressed to Ramkrishna S. Solanki, ramkssolanki@gmail.com
}

Received 28 February 2012; Accepted 29 March 2012

Academic Editors: J. Hu and J. López-Fidalgo

Copyright (c) 2012 Ramkrishna S. Solanki et al. This is an open access article distributed under the Creative Commons Attribution License, which permits unrestricted use, distribution, and reproduction in any medium, provided the original work is properly cited.

This paper suggests a class of estimators for estimating the finite population mean $\bar{Y}$ of the study variable $y$ using known population mean $\bar{X}$ of the auxiliary variable $x$. Asymptotic expressions of bias and variance of the suggested class of estimators have been obtained. Asymptotic optimum estimator (AOE) in the class is identified along with its variance formula. It has been shown that the proposed class of estimators is more efficient than usual unbiased, usual ratio, usual product, Bahl and Tuteja (1991), and Kadilar and Cingi (2003) estimators under some realistic conditions. An empirical study is carried out to judge the merits of suggested estimator over other competitors practically.

\section{Introduction}

The literature on survey sampling describes a great variety of techniques for using auxiliary information to obtain more efficient estimators. Ratio, product, and regression methods of estimation are good examples in this context (see Singh [1]). If the correlation between the study variable $y$ and the auxiliary variable $x$ is positive (high), the ratio method of estimation is quite effective. On the other hand, if this correlation is negative (high) the product method of estimation envisaged by Robson [2] and rediscovered by Murthy [3] can be employed quite effectively. The classical ratio and product estimators are considered to be the most practicable in many situations, but they have the limitation of having at most the same efficiency as that of the linear regression estimator. In the situation where the relation between the study variable $y$ and the auxiliary variable $x$ is a straight line and passing through the origin, the ratio and product estimators have efficiencies equal to the usual regression estimator. But in many practical situations, the line does not pass through the origin, and in such 
circumstances the ratio and product estimators do not perform equally well to the regression estimator. Keeping this fact in view, a large number of authors have paid their attention toward the formulation of modified ratio and product estimators, for instance, see Singh $[4,5]$, Srivastava [6-8], Reddy [9, 10], Gupta [11], Sahai [12], Sahai and Ray [13], Ray and Sahai [14], Srivenkataramana and Tracy [15, 16], Bandyopadhyay [17], Vos [18], Sisodia and Dwivedi [19], Adhvaryu and Gupta [20], Chaubey et al. [21], Mohanty and Sahoo [22], Singh and Shukla [23], Naik and Gupta [24], Bahl and Tuteja [25], Mohanty and Sahoo [26], Upadhyaya and Singh [27], Singh and Tailor [28], Kadilar and Cingi [29], Singh et al. [30], and others.

In this paper, we have envisaged a new class of estimators for population mean of study variable $y$ using information on an auxiliary variable $x$ which is highly correlated with the study variable and have shown that the suggested class of estimators is more efficient than some existing estimators.

Consider a finite population $U=\left(u_{1}, u_{2}, \ldots, u_{N}\right)$ of size $N$ from which a sample of size $n$ is drawn according to simple random sampling without replacement (SRSWOR). Let $\bar{y}$ and $\bar{x}$ be the sample mean estimators of the population means $\bar{Y}$ and $\bar{X}$, respectively, of the study variable $y$ and the auxiliary variable $x$. Let $\left.C_{y}\left(=S_{y} / \bar{Y}\right)\right), C_{x}\left(=S_{x} / \bar{X}\right), \rho\left(=S_{y x} / S_{x} S_{y}\right)$ (correlation coefficient between the variables $y$ and $x$ ) and $k=\left(\rho C_{y} / C_{x}\right)$, where

$$
\begin{gathered}
S_{y}^{2}=(N-1)^{-1} \sum_{i=1}^{N}\left(y_{i}-\bar{Y}\right)^{2}, \quad S_{x}^{2}=(N-1)^{-1} \sum_{i=1}^{N}\left(x_{i}-\bar{X}\right)^{2}, \\
S_{x y}=(N-1)^{-1} \sum_{i=1}^{N}\left(x_{i}-\bar{X}\right)\left(y_{i}-\bar{Y}\right) .
\end{gathered}
$$

The remaining part of the paper is organized as follows. Section 2 gives the brief review of some estimators for the population mean of study variable $y$ with its properties. In Section 3, a new class of estimators for the population mean is described, and the expressions for the asymptotic bias and variance are obtained. Asymptotic optimum estimator (AOE) in the suggested class is obtained with its variance formula. Section 4 addresses the problem of efficiency comparisons, while in Section 5 an empirical study is carried out to evaluate the performance of different estimators.

\section{Reviewing Estimators}

It is very well known that the sample mean $\bar{y}$ is an unbiased estimator of population mean $\bar{Y}$, and under (SRSWOR), its variance is given by

$$
\operatorname{Var}(\bar{y})=\frac{(1-f)}{n} S_{y}^{2}=\frac{(1-f)}{n} \bar{Y}^{2} C_{y^{\prime}}^{2}
$$

where $f=(n / N)$. 
The usual ratio and product estimators of population mean $\bar{Y}$ of the study variable $y$ are, respectively, defined as

$$
\begin{aligned}
& \bar{y}_{R}=\bar{y} \frac{\bar{X}}{\bar{x}}, \\
& \bar{y}_{P}=\bar{y} \frac{\bar{x}}{\bar{X}} .
\end{aligned}
$$

Bahl and Tuteja [25] suggested exponential ratio-type and product-type estimators for population mean $\bar{Y}$, respectively, as

$$
\begin{aligned}
& \bar{y}_{\mathrm{Re}}=\bar{y} \exp \left(\frac{\bar{X}-\bar{x}}{\bar{X}+\bar{x}}\right), \\
& \bar{y}_{\mathrm{Pe}}=\bar{y} \exp \left(\frac{\bar{x}-\bar{X}}{\bar{x}+\bar{X}}\right) .
\end{aligned}
$$

as

Kadilar and Cingi [31] suggested a chain ratio-type estimator for population mean $\bar{Y}$

$$
\bar{y}_{\mathrm{CR}}=\bar{y}\left(\frac{\bar{X}^{2}}{\bar{x}^{2}}\right) .
$$

Following the procedure adopted by Kadilar and Cingi [31], one may define a chain product-type estimator for population mean $\bar{Y}$ as

$$
\bar{y}_{\mathrm{CP}}=\bar{y}\left(\frac{\bar{x}^{2}}{\bar{X}^{2}}\right) .
$$

To the first degree of approximation, the biases and variances of estimators $\bar{y}_{R^{\prime}}, \bar{y}_{p^{\prime}}, \bar{y}_{\mathrm{Re}^{\prime}}, \bar{y}_{\mathrm{Pe}^{\prime}}$ $\bar{y}_{\mathrm{CR}}$, and $\bar{y}_{\mathrm{CP}}$ are, respectively, given by

$$
\begin{gathered}
B\left(\bar{y}_{R}\right)=\frac{(1-f)}{n} \bar{Y} C_{x}^{2}(1-k), \\
B\left(\bar{y}_{P}\right)=\frac{(1-f)}{n} \bar{Y} C_{x}^{2} k, \\
B\left(\bar{y}_{\operatorname{Re}}\right)=\frac{(1-f)}{n} C_{x}^{2}\left(\frac{\bar{Y}}{8}\right)(3-4 k),
\end{gathered}
$$




$$
\begin{gathered}
B\left(\bar{y}_{\mathrm{Pe}}\right)=\frac{(1-f)}{n} C_{x}^{2}\left(\frac{\bar{Y}}{8}\right)(4 k-1), \\
B\left(\bar{y}_{\mathrm{CR}}\right)=\frac{(1-f)}{n} \bar{Y} C_{x}^{2}(3-2 k), \\
B\left(\bar{y}_{\mathrm{CP}}\right)=\frac{(1-f)}{n} \bar{Y} C_{x}^{2}(1+2 k), \\
\operatorname{Var}\left(\bar{y}_{R}\right)=\frac{(1-f)}{n} \bar{Y}^{2}\left[C_{y}^{2}+C_{x}^{2}(1-2 k)\right], \\
\operatorname{Var}\left(\bar{y}_{P}\right)=\frac{(1-f)}{n} \bar{Y}^{2}\left[C_{y}^{2}+C_{x}^{2}(1+2 k)\right], \\
\operatorname{Var}\left(\bar{y}_{\mathrm{Re}}\right)=\frac{(1-f)}{n} \bar{Y}^{2}\left[C_{y}^{2}+\frac{C_{x}^{2}}{4}(1-4 k)\right], \\
\operatorname{Var}\left(\bar{y}_{\mathrm{Pe}}\right)=\frac{(1-f)}{n} \bar{Y}^{2}\left[C_{y}^{2}+\frac{C_{x}^{2}}{4}(1+4 k)\right], \\
\operatorname{Var}\left(\bar{y}_{\mathrm{CR}}\right)=\frac{(1-f)}{n} \bar{Y}^{2}\left[C_{y}^{2}+4 C_{x}^{2}(1-k)\right], \\
\operatorname{Var}\left(\bar{y}_{\mathrm{CP}}\right)=\frac{(1-f)}{n} \bar{Y}^{2}\left[C_{y}^{2}+4 C_{x}^{2}(1+k)\right] .
\end{gathered}
$$

From (2.1) and (2.12)-(2.17), we have made some efficiency comparisons between the estimators $\bar{y}, \bar{y}_{R}, \bar{y}_{p}, \bar{y}_{\mathrm{Re}^{\prime}}, \bar{y}_{\mathrm{Pe}}, \bar{y}_{\mathrm{CR}}$, and $\bar{y}_{\mathrm{CP}}$, as shown in Table 1 .

\section{Suggested Class of Estimators}

We define the following class of estimators for the population mean $\bar{Y}$ as

$$
t_{(\alpha, \delta)}=\bar{y}\left[2-\left(\frac{\bar{x}}{\bar{X}}\right)^{\alpha} \exp \left\{\frac{\delta(\bar{x}-\bar{X})}{(\bar{x}+\bar{X})}\right\}\right]
$$

where $(\alpha, \delta)$ are suitable chosen scalars.

It is to be mentioned that for $\delta=0$, the class of estimators $t_{(\alpha, \delta)}$ reduces to the following class of estimators

$$
t_{(\alpha, 0)}=\bar{y}\left[2-\left(\frac{\bar{x}}{\bar{X}}\right)^{\alpha}\right]
$$

which is due to Sahai and Ray [13]. 
Table 1: Efficiency comparisons between different estimators.

\begin{tabular}{lcc}
\hline Estimator & More efficient than estimator & If \\
\hline $\bar{y}$ & $\bar{y}_{R}$ & $k \leq 1 / 2$ \\
$\bar{y}$ & $\bar{y}_{P}$ & $k \geq-1 / 2$ \\
$\bar{y}_{R}$ & $\bar{y}$ & $k>1 / 2$ \\
$\bar{y}_{P}$ & $\bar{y}$ & $k<-1 / 2$ \\
$\bar{y}$ & $\bar{y}_{\mathrm{Re}}$ & $k \leq 1 / 4$ \\
$\bar{y}$ & $\bar{y}_{\mathrm{Pe}}$ & $k \geq-1 / 4$ \\
$\bar{y}_{\mathrm{Re}}$ & $\bar{y}_{\mathrm{y}}$ & $k>1 / 4$ \\
$\bar{y}_{\mathrm{Pe}}$ & $\bar{y}$ & $k<-1 / 4$ \\
$\bar{y}$ & $\bar{y}_{\mathrm{CR}}$ & $k \leq 1$ \\
$\bar{y}$ & $\bar{y}_{\mathrm{CP}}$ & $k \geq-1$ \\
$\bar{y}_{\mathrm{CR}}$ & $\bar{y}_{\mathrm{y}}$ & $k>1$ \\
$\bar{y}_{\mathrm{CP}}$ & $\bar{y}_{\mathrm{y}}$ & $k<-1$ \\
$\bar{y}_{\mathrm{CR}}$ & $\bar{y}_{R}$ & $k>3 / 2$ \\
$\bar{y}_{\mathrm{CP}}$ & $\bar{y}_{P}$ & $k<-3 / 2$ \\
\hline & &
\end{tabular}

While for $\alpha=0$, it reduces to the new transformed class of estimators defined as

$$
t_{(0, \delta)}=\bar{y}\left[2-\exp \left\{\frac{\delta(\bar{x}-\bar{X})}{(\bar{x}+\bar{X})}\right\}\right] .
$$

To obtain the bias and variance of suggested class of estimators $t_{(\alpha, \delta)}$, we write

$$
\begin{aligned}
& \bar{y} c=\bar{Y}\left(1+e_{0}\right), \\
& \bar{x}_{C}=\bar{X}\left(1+e_{1}\right),
\end{aligned}
$$

such that

$$
\begin{gathered}
E\left(e_{0}\right)=E\left(e_{1}\right)=0, \\
E\left(e_{0}^{2}\right)=\frac{(1-f)}{n} C_{y^{\prime}}^{2} \\
E\left(e_{1}^{2}\right)=\frac{(1-f)}{n} C_{x^{\prime}}^{2} \\
E\left(e_{0} e_{1}\right)=\frac{(1-f)}{n} \rho C_{y} C_{x}=\frac{(1-f)}{n} k C_{x}^{2} .
\end{gathered}
$$


Expressing (3.1) in terms of $e^{\prime}$ s, we have

$$
\begin{aligned}
t_{(\alpha, \delta)} & =\bar{Y}\left(1+e_{0}\right)\left[2-\left(1+e_{1}\right)^{\alpha} \exp \left\{\frac{\delta e_{1}}{2+e_{1}}\right\}\right] \\
& =\bar{Y}\left(1+e_{0}\right)\left[2-\left(1+e_{1}\right)^{\alpha} \exp \left\{\frac{\delta e_{1}}{2}\left(1+\frac{e_{1}}{2}\right)^{-1}\right\}\right]
\end{aligned}
$$

We assume that $\left|e_{1}\right|<1$, so that $\left(1+e_{1}\right)^{\alpha}, \exp \left\{\left(\delta e_{1} / 2\right)\left(1+e_{1} / 2\right)^{-1}\right\}$, and $\left(1+e_{1} / 2\right)^{-1}$ are expandable. Now expanding the right-hand side of (3.6), we have

$$
\begin{aligned}
t_{(\alpha, \delta)=} \bar{Y}\left(1+e_{0}\right)\left[2-\left\{1+\alpha e_{1}+\frac{\alpha(\alpha-1)}{2} e_{1}^{2}+\cdots\right\}\right. \\
\left.\quad \times\left\{1+\frac{\delta e_{1}}{2}\left(1+\frac{e_{1}}{2}\right)^{-1}+\frac{\delta^{2} e_{1}^{2}}{8}\left(1+\frac{e_{1}}{2}\right)^{-2}+\cdots\right\}\right] \\
=\bar{Y}\left(1+e_{0}\right)\left[2-\left\{1+\alpha e_{1}+\frac{\alpha(\alpha-1)}{2} e_{1}^{2}+\cdots\right\}\right. \\
\left.\quad \times\left\{1+\frac{\delta e_{1}}{2}\left(1-\frac{e_{1}}{2}+\frac{e_{1}^{2}}{4}-\cdots+\frac{\delta^{2} e_{1}^{2}}{8}\left(1-e_{1}\right)+\cdots\right)\right\}\right] \\
=\bar{Y}\left(1+e_{0}\right)\left[2-\left\{1+\frac{(2 \alpha+\delta)}{2} e_{1}+\frac{(2 \alpha+\delta)(2 \delta+\delta-2)}{8} e_{1}^{2}+\cdots\right\}\right] \\
=\bar{Y}\left[\left\{1+e_{0}-\frac{(2 \alpha+\delta)}{2}\left(e_{1}+e_{0} e_{1}\right)-\frac{(2 \alpha+\delta)(2 \delta+\delta-2)}{8}\left(e_{1}^{2}+e_{0} e_{1}^{2}\right)-\cdots\right\}\right] .
\end{aligned}
$$

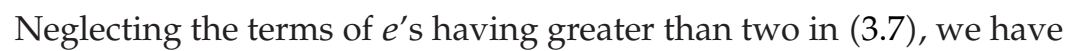

$$
t_{(\alpha, \delta)} \cong \bar{Y}\left[1+e_{0}-\frac{(2 \alpha+\delta)}{2}\left\{e_{1}+e_{0} e_{1}-\frac{(2 \delta+\delta-2)}{4} e_{1}^{2}\right\}\right]
$$

or

$$
\left(t_{(\alpha, \delta)}-\bar{Y}\right) \cong \bar{Y}\left[e_{0}-\frac{(2 \alpha+\delta)}{2}\left\{e_{1}+e_{0} e_{1}-\frac{(2 \delta+\delta-2)}{4} e_{1}^{2}\right\}\right]
$$

Taking expectation of both sides of (3.9), we get the bias of the class of estimators $t_{(\alpha, \delta)}$ to the first degree of approximation as

$$
B\left(t_{(\alpha, \delta)}\right)=\frac{(1-f)}{n} \bar{Y} C_{x}^{2}\left[-\frac{(2 \alpha+\delta)}{2}\left\{\frac{(2 \delta+\delta-2)}{4}+k\right\}\right] .
$$

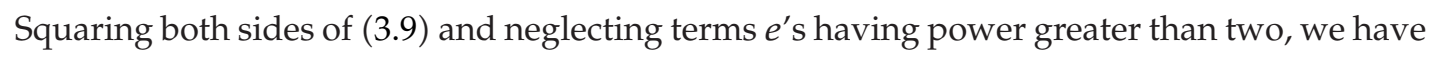

$$
\left(t_{(\alpha, \delta)}-\bar{Y}\right)^{2} \cong \bar{Y}\left[e_{0}^{2}+\frac{(2 \alpha+\delta)}{2}\left\{\frac{(2 \delta+\delta)}{4} e_{1}^{2}-2 e_{0} e_{1}\right\}\right]
$$


Taking expectation of both sides of (3.11), we get the variance of $t_{(\alpha, \delta)}$ to the first degree of approximation as

$$
\operatorname{Var}\left(t_{(\alpha, \delta)}\right)=\frac{(1-f)}{n} \bar{Y}^{2}\left[C_{y}^{2}+\frac{(2 \alpha+\delta)}{4} C_{x}^{2}\{(2 \alpha+\delta)-4 k\}\right]
$$

which is minimum when

$$
(2 \alpha+\delta)=2 k
$$

Thus, the resulting minimum variance of $t_{(\alpha, \delta)}$ is obtained as

$$
\operatorname{Var}_{\min }\left(t_{(\alpha, \delta)}\right)=\frac{(1-f)}{n} S_{y}^{2}\left(1-\rho^{2}\right)
$$

The minimum variance of $t_{(\alpha, \delta)}$ equals to the approximate variance of the usual linear regression estimator defined as

$$
\bar{y}_{\mathrm{lr}}=\bar{y}+\widehat{\beta}(\bar{X}-\bar{x})
$$

where $\widehat{\beta}=\left(s_{x y} / s_{x}^{2}\right), s_{x y}=(n-1)^{-1} \sum_{i=1}^{n}\left(x_{i}-\bar{x}\right)\left(y_{i}-\bar{y}\right)$ and $s_{x}^{2}=(n-1)^{-1} \sum_{i=1}^{n}\left(x_{i}-\bar{x}\right)^{2}$.

It is interesting to note that if we set $(\delta=0)$ and $(\alpha=0)$ in (3.12), we get the variances of the classes of estimators $t_{(\alpha, 0)}$ and $t_{(0, \delta)}$, respectively, as

$$
\begin{gathered}
\operatorname{Var}\left(t_{(\alpha, 0)}\right)=\frac{(1-f)}{n} \bar{Y}^{2}\left[C_{y}^{2}+\alpha C_{x}^{2}(\alpha-2 k)\right], \\
\operatorname{Var}\left(t_{(0, \delta)}\right)=\frac{(1-f)}{n} \bar{Y}^{2}\left[C_{y}^{2}+\left(\frac{\delta}{4}\right) C_{x}^{2}(\delta-4 k)\right] .
\end{gathered}
$$

If we assume the value of $\alpha$ is specified by $\alpha_{o}$ (say), then the variances of $t_{(\alpha, 0)}$ and $t_{(\alpha, \delta)}$ are, respectively, given by

$$
\begin{gathered}
\operatorname{Var}\left(t_{\left(\alpha=\alpha_{0}, 0\right)}\right)=\frac{(1-f)}{n} \bar{Y}^{2}\left[C_{y}^{2}+\alpha_{o} C_{x}^{2}\left(\alpha_{o}-2 k\right)\right] \\
\operatorname{Var}\left(t_{\left(\alpha=\alpha_{o}, \delta\right)}\right)=\frac{(1-f)}{n} \bar{Y}^{2}\left[C_{y}^{2}+\frac{\left(2 \alpha_{o}+\delta\right)}{4} C_{x}^{2}\left\{\left(2 \alpha_{o}+\delta\right)-4 k\right\}\right] .
\end{gathered}
$$

If the value of $\delta$ is specified $\delta_{o}$, then the variances of the estimators $t_{(0, \delta)}$ and $t_{(\alpha, \delta)}$ are, respectively, given by

$$
\begin{gathered}
\operatorname{Var}\left(t_{\left(0, \delta=\delta_{o}\right)}\right)=\frac{(1-f)}{n} \bar{Y}^{2}\left[C_{y}^{2}+\left(\frac{\delta_{o}}{4}\right) C_{x}^{2}\left(\delta_{o}-4 k\right)\right] \\
\operatorname{Var}\left(t_{\left(\alpha, \delta=\delta_{o}\right)}\right)=\frac{(1-f)}{n} \bar{Y}^{2}\left[C_{y}^{2}+\frac{\left(2 \alpha_{o}+\delta\right)}{4} C_{x}^{2}\left\{\left(2 \alpha+\delta_{o}\right)-4 k\right\}\right]
\end{gathered}
$$


To illustrate our general results, we consider a particular case of the proposed class of estimators $t_{(\alpha, \delta)}$ with its properties. If we set $(\alpha, \delta)=(1,1)$ in $(3.1)$, we get an estimator of population mean $\bar{Y}$ as

$$
t_{(1,1)}=\bar{y}\left[2-\left(\frac{\bar{x}}{\bar{X}}\right) \exp \left(\frac{\bar{x}-\bar{X}}{\bar{x}+\bar{X}}\right)\right]
$$

Putting $(\alpha, \delta)=(1,1)$ in (3.10) and (3.12), we get the bias and variance of $t_{(1,1)}$, to the first degree of approximation, respectively, as

$$
\begin{gathered}
B\left(t_{(1,1)}\right)=-\frac{3(1-f)}{8 n} \bar{Y} C_{x}^{2}(1+4 K), \\
\operatorname{Var}\left(t_{(1,1)}\right)=\frac{(1-f)}{n} \bar{Y}^{2}\left[C_{y}^{2}+\frac{3 C_{x}^{2}}{4}(3-4 k)\right] .
\end{gathered}
$$

Expression (3.20) clearly indicates that the proposed estimator $t_{(1,1)}$ is better than conventional unbiased estimator $\bar{y}$ if $k>(3 / 4)$, a condition which is usually met in survey situations.

\section{Efficiency Comparisons}

From (3.17), we have

$$
\begin{aligned}
& \operatorname{Var}\left(t_{\left(\alpha=\alpha_{o}, \delta\right)}\right)-\operatorname{Var}\left(t_{\left(\alpha=\alpha_{o}, 0\right)}\right) \\
& =\frac{(1-f)}{n} \bar{Y}^{2} C_{x}^{2}\left[\frac{\left(2 \alpha_{o}+\delta\right)}{4}\left\{\left(2 \alpha_{o}+\delta\right)-4 k\right\}-\alpha_{o}\left(\alpha_{o}-2 k\right)\right] \\
& <0, \quad \text { if }\left[\left(2 \alpha_{o}+\delta\right)^{2}-4\left(2 \alpha_{o}+\delta\right) k-4 \alpha_{o}^{2}+8 \alpha_{o} k\right]<0, \\
& \text { i.e., } \quad \text { if } \delta\left(\delta+4 \alpha_{o}-4 k\right)<0, \\
& \text { i.e., } \quad \text { if } \begin{cases}\text { either } & 0<\delta<4\left(k-\alpha_{o}\right) \\
\text { or } & 4\left(k-\alpha_{o}\right)<\delta<0,\end{cases}
\end{aligned}
$$

or equivalently

$$
\min \cdot\left\{0,4\left(k-\alpha_{o}\right)\right\}<\delta<\max \cdot\left\{0,4\left(k-\alpha_{o}\right)\right\} .
$$


From (3.18), we have

$$
\begin{aligned}
& \operatorname{Var}\left(t_{\left(\alpha, \delta=\delta_{o}\right)}\right)-\operatorname{Var}\left(t_{\left(0, \delta_{o}\right)}\right) \\
& \left.=\frac{(1-f)}{n} \bar{Y}^{2} C_{x}^{2}\left[\frac{\left(2 \alpha+\delta_{o}\right)^{2}}{4}-\left(2 \alpha+\delta_{o}\right) k-\frac{\delta_{o}^{2}}{4}+\delta_{o} k\right\}\right] \\
& <0, \quad \text { if }\left[\alpha^{2}+\alpha \delta_{o}-2 \alpha k\right]<0, \\
& \text { i.e., if } \begin{cases}\text { either } & 0<\alpha<\left(2 k-\delta_{o}\right) \\
\text { or } & \left(2 k-\delta_{o}\right)<\alpha<0,\end{cases}
\end{aligned}
$$

or equivalently

$$
\min \cdot\left\{0,\left(2 k-\delta_{o}\right)\right\}<\alpha<\max \cdot\left\{0,\left(2 k-\delta_{o}\right)\right\} .
$$

From (2.1), (2.12)-(2.17), and (3.12), we have

(i)

$$
\begin{aligned}
& \operatorname{Var}\left(t_{(\alpha, \delta)}\right)-\operatorname{Var}(\bar{y}) \\
& \quad=\frac{(1-f)}{4 n} \bar{Y}^{2} C_{x}^{2}\left[(2 \alpha+\delta)^{2}-k(2 \alpha+\delta)\right] \\
& <0, \quad \text { if } \begin{cases}\text { either } & 0<(2 \alpha+\delta)<k \\
\text { or } & k<(2 \alpha+\delta)<0,\end{cases}
\end{aligned}
$$

or equivalently

$$
\min \cdot(0, k)<(2 \alpha+\delta)<\max \cdot(0, k) .
$$

(ii)

$$
\begin{aligned}
& \operatorname{Var}\left(t_{(\alpha, \delta)}\right)-\operatorname{Var}\left(\bar{y}_{R}\right) \\
& \quad=\frac{(1-f)}{n} \bar{Y}^{2} C_{x}^{2}\left[\frac{(2 \alpha+\delta)}{4}\{(2 \alpha+\delta)-4 k\}-(1+2 k)\right] \\
& <0, \quad \text { if } \begin{cases}\text { either } & 2<(2 \alpha+\delta)<2(2 k-1) \\
\text { or } & 2(2 k-1)<(2 \alpha+\delta)<2,\end{cases}
\end{aligned}
$$

or equivalently

$$
\min \cdot\{2,2(2 k-1)\}<(2 \alpha+\delta)<\max \cdot\{2,2(2 k-1)\} .
$$


(iii)

$$
\begin{aligned}
& \operatorname{Var}\left(t_{(\alpha, \delta)}\right)-\operatorname{Var}\left(\bar{y}_{p}\right) \\
& =\frac{(1-f)}{n} \bar{Y}^{2} C_{x}^{2}\left[\frac{(2 \alpha+\delta)}{4}\{(2 \alpha+\delta)-4 k\}-(1+2 k)\right] \\
& <0, \quad \text { if } \begin{cases}\text { either } & -2<\theta<2(2 k+1) \\
\text { or } & 2(2 k+1)<\theta<-2,\end{cases}
\end{aligned}
$$

or equivalently

$$
\min \cdot\{-2,2(2 k+1)\}<(2 \alpha+\delta)<\max \cdot\{-2,2(2 k+1)\} .
$$

(iv)

$$
\begin{aligned}
& \operatorname{Var}\left(t_{(\alpha, \delta)}\right)-\operatorname{Var}\left(\bar{y}_{\mathrm{Re}}\right) \\
& \quad=\frac{(1-f)}{4 n} \bar{Y}^{2} C_{x}^{2}[(2 \alpha+\delta)\{(2 \alpha+\delta)-4 k\}-(1-4 k)] \\
& <0, \quad \text { if } \begin{cases}\text { either } & 1<(2 \alpha+\delta)<(4 k-1) \\
\text { or } & (4 k-1)<(2 \alpha+\delta)<1,\end{cases}
\end{aligned}
$$

or equivalently

$$
\min \cdot\{1,(4 k-1)\}<(2 \alpha+\delta)<\max \cdot\{1,(4 k-1)\}
$$

( v)

$$
\begin{aligned}
& \operatorname{Var}\left(t_{(\alpha, \delta)}\right)-\operatorname{Var}\left(\bar{y}_{\mathrm{Pe}}\right) \\
& \quad=\frac{(1-f)}{4 n} \bar{Y}^{2} C_{x}^{2}[(2 \alpha+\delta)\{(2 \alpha+\delta)-4 k\}-(1+4 k)] \\
& <0, \quad \text { if } \begin{cases}\text { either } & -1<(2 \alpha+\delta)<(4 k+1) \\
\text { or } & (4 k+1)<(2 \alpha+\delta)<-1,\end{cases}
\end{aligned}
$$

or equivalently

$$
\min \cdot\{-1,(4 k+1)\}<(2 \alpha+\delta)<\max \cdot\{-1,(4 k+1)\}
$$


(vi)

$$
\begin{aligned}
& \operatorname{Var}\left(t_{(\alpha, \delta)}\right)-\operatorname{Var}\left(\bar{y}_{\mathrm{CR}}\right) \\
& \quad=\frac{(1-f)}{n} \bar{Y}^{2} C_{x}^{2}\left[\frac{(2 \alpha+\delta)}{4}\{(2 \alpha+\delta)-4 k\}-4(1-k)\right] \\
& \quad<0, \quad \text { if } \begin{cases}\text { either } & 4<(2 \alpha+\delta)<4(k-1) \\
\text { or } & 4(k-1)<(2 \alpha+\delta)<4,\end{cases}
\end{aligned}
$$

or equivalently

$$
\min \cdot\{4,4(k-1)\}<(2 \alpha+\delta)<\max \cdot\{4,4(k-1)\}
$$

(vii)

$$
\begin{aligned}
& \operatorname{Var}\left(t_{(\alpha, \delta)}\right)-\operatorname{Var}\left(\bar{y}_{\mathrm{CP}}\right) \\
& \quad=\frac{(1-f)}{n} \bar{Y}^{2} C_{x}^{2}\left[\frac{(2 \alpha+\delta)}{4}\{(2 \alpha+\delta)-4 k\}-4(1+k)\right] \\
& \quad<0, \quad \text { if } \begin{cases}\text { either } & -4<(2 \alpha+\delta)<4(k+1) \\
\text { or } & 4(k+1)<(2 \alpha+\delta)<-4,\end{cases}
\end{aligned}
$$

or equivalently

$$
\min \cdot\{-4,4(k+1)\}<(2 \alpha+\delta)<\max \cdot\{-4,4(k+1)\}
$$

From (2.1), (2.12), (2.14), (2.16), and (3.21), we have made some efficiency comparisons between the estimators $t_{(1,1)}, \bar{y}, \bar{y}_{R}, \bar{y}_{\mathrm{Re}^{\prime}}$ and $\bar{y}_{\mathrm{CR}}$, as shown in Table 2 .

It is clearly indicated from Table 2 that the estimator $t_{(1,1)}$ is more efficient than estimators $\bar{y}, \bar{y}_{R}, \bar{y}_{\mathrm{Re}^{\prime}}$ and $\bar{y}_{\mathrm{CR}}$ if

$$
\frac{5}{4}<k<\frac{7}{3}
$$

\section{Empirical Study}

To judge the merits of the suggested estimator $t_{(1,1)}$ over usual unbiased estimator $\bar{y}$, usual ratio estimator $\bar{y}_{R}$, Bahl and Tuteja [25] exponential ratio-type estimator $\bar{y}_{R e}$ and Kadilar and Cingi [31] chain ratio-type estimator $\bar{y}_{\mathrm{CR}}$ we have considered three populations. Descriptions of the populations are given below. 
Table 2: Efficiency comparisons between different estimators.

\begin{tabular}{lcc}
\hline Estimator & More efficient than estimator & If \\
\hline$t_{(1,1)}$ & $\bar{y}$ & $k>3 / 4$ \\
$t_{(1,1)}$ & $\bar{y}_{R}$ & $k>5 / 4$ \\
$t_{(1,1)}$ & $\bar{y}_{\operatorname{Re}}$ & $k>1$ \\
$t_{(1,1)}$ & $\bar{y}_{\mathrm{CR}}$ & $k<7 / 3$ \\
\hline
\end{tabular}

Table 3: PREs of different estimators of population mean $\bar{y}$.

\begin{tabular}{lccc}
\hline \multirow{2}{*}{ Estimator } & \multicolumn{3}{c}{ PRE $(\cdot, \bar{y})$ for population } \\
& I & II & III \\
\hline $\bar{y}$ (sample mean) & 100.00 & 100.00 & 100.00 \\
$\bar{y}_{R}$ (ratio estimator) & 173.46 & 226.77 & 451.25 \\
$\bar{y}_{\text {Re }}$ (bahl and Tuteja [25]) & 133.16 & 151.03 & 199.15 \\
$\bar{y}_{\mathrm{CR}}$ (kadilar and Cingi [31]) & 221.83 & 286.48 & 320.12 \\
$t_{(1,1)}$ (proposed estimator) & $\mathbf{2 2 4 . 6 4}$ & $\mathbf{2 9 7 . 0 5}$ & $\mathbf{6 3 1 . 5 9}$ \\
\hline
\end{tabular}

Population I (source: Srivastava [7], page 406)

$y$ : fiber per plant in jute fiber crops,

$x$ : height,

$C_{y}^{2}=0.0568, C_{x}^{2}=0.00846, \rho=0.7418, k=1.9221$.

Population II (source: Gupta and Shabbir [32])

$y$ : the level of apple production amount ( 1 unit $=100$ tones),

$x$ : the number of apple trees ( 1 unit $=100$ trees),

$C_{y}^{2}=17.4724, C_{x}^{2}=4.0804, \rho=0.82, k=1.6968$.

Population III (Source: Kadilar and Cingi [33])

$y$ : the level of apple production amount,

$x$ : the number of apple trees,

$C_{y}^{2}=34.1056, C_{x}^{2}=14.8225, \rho=0.92, k=1.3955$.

We have computed the percent relative efficiencies (PREs) of different estimators $(\cdot) \bar{y}$, $\bar{y}_{R}, \bar{y}_{\mathrm{Re}}, \bar{y}_{\mathrm{CR}}$, and $t_{(1,1)}$ of the population mean $\bar{Y}$ with respect to $\bar{y}$ by using the following formula:

$$
\operatorname{PRE}(\cdot, \bar{y})=\frac{\operatorname{Var}(\bar{y})}{\operatorname{Var}(\cdot)} \times 100,
$$

and results are summarized in Table 3.

Table 3 exhibits that the proposed estimator $t_{(1,1)}$ is more efficient than usual unbiased estimator $\bar{y}$, usual ratio estimator $\bar{y}_{R^{\prime}}$ Bahl and Tuteja [25] estimator $\bar{y}_{\mathrm{Re}^{\prime}}$, and Kadilar and 
Cingi [31] estimator $\bar{y}_{C R}$ in the sense of having the largest PRE in all three populations. In populations II and III, the proposed estimator $t_{(1,1)}$ has the largest gain in efficiency over all the estimators, while in population I, there is marginal gain in efficiency as compared to $\bar{y}_{\mathrm{CR}}$. It is further noted that the condition $5 / 4<k<7 / 4$ has been satisfied in population I $(1.25<$ $1.9221<2.33)$, II $(1.25<1.6968<2.33)$, and III $(1.25<1.3955<2.33)$. Thus, we recommend the proposed estimator $t_{(1,1)}$ for its use in practice wherever the condition $5 / 4<k<7 / 4$ is satisfied.

\section{References}

[1] S. Singh, Advanced Sampling Theory with Applications. Vol. I, II, Kluwer Academic, Dordrecht, The Netherlands, 2003.

[2] D.S. Robson, "Applications of multivariate polykays to the theory of unbiased ratio-type estimation," Journal of the American Statistical Association, vol. 52, pp. 511-522, 1957.

[3] M. N. Murthy, "Product method of estimation," Sankhya, vol. 26, pp. 69-74, 1964.

[4] M. P. Singh, "On the estimation of ratio and product of the population parameters," Sankhya, vol. 27, pp. 321-328, 1965.

[5] M. P. Singh, "Ratio cum product method of estimation," Metrika, vol. 12, pp. 34-42, 1967.

[6] S. K. Srivastava, "An estimator using auxiliary information in sample surveys," Bulletin/Calcutta Statistical Association Bulletin, vol. 16, pp. 121-132, 1967.

[7] S. K. Srivastava, "A generalized estimator for the mean of a finite population using multi auxiliary information," Journal of the American Statistical Association, vol. 66, pp. 404-407, 1971.

[8] S. K. Srivastava, "A class of estimators using auxiliary information in sample surveys," The Canadian Journal of Statistics, vol. 8, no. 2, pp. 253-254, 1980.

[9] V. N. Reddy, "On ratio and product methods of estimation," Sankhya B, vol. 35, no. 3, pp. 307-316, 1973.

[10] V. N. Reddy, "On transformed ratio method of estimation," Sankhya C, vol. 36, pp. 59-70, 1974.

[11] P. C. Gupta, "On some quadratic and higher degree ratio and product estimators," Journal of the Indian Society of Agricultural Statistics, vol. 30, no. 2, pp. 71-80, 1978.

[12] A. Sahai, "An efficient variant of the product and ratio estimators," Statistica Neerlandica, vol. 33, no. 1, pp. 27-35, 1979.

[13] A. Sahai and S. K. Ray, "An efficient estimator using auxiliary information," Metrika, vol. 27, no. 4, pp. 271-275, 1980.

[14] S. K. Ray and A. Sahai, "Efficient families of ratio and product-type estimators," Biometrika, vol. 67, no. 1, pp. 211-215, 1980.

[15] T. Srivenkataramana and D. S. Tracy, "An alternative to ratio method in sample surveys," Annals of the Institute of Statistical Mathematics, vol. 32, no. 1, pp. 111-120, 1980.

[16] T. Srivenkataramana and D. S. Tracy, "Extending product method of estimation to positive correlation case in surveys," The Australian Journal of Statistics, vol. 23, no. 1, pp. 93-100, 1981.

[17] S. Bandyopadhyay, "Improved ratio and product estimators," Sankhya, vol. 42, no. 1-2, pp. 45-49, 1980.

[18] J. W. E. Vos, "Mixing of direct, ratio, and product method estimators," Statistica Neerlandica, vol. 34, no. 4, pp. 209-218, 1980.

[19] B. V. S. Sisodia and V. K. Dwivedi, "A modified ratio estimator using coefficient of an auxiliary variable," Journal of the Indian Society of Agricultural Statistics, vol. 33, no. 2, pp. 13-18, 1981.

[20] D. Adhvaryu and P. C. Gupta, "On some alternative sampling strategies using auxiliary information," Metrika, vol. 30, no. 4, pp. 217-226, 1983.

[21] Y. P. Chaubey, T. D. Dwivedi, and M. Singh, "An efficiency comparison of product and ratio estimator," Communications in Statistics. A. Theory and Methods, vol. 13, no. 6, pp. 699-709, 1984.

[22] S. Mohanty and L. N. Sahoo, "A class of estimators based on mean per unit and ratio estimators," Statistica, vol. 47, no. 3, pp. 473-477, 1987.

[23] V. K. Singh and D. Shukla, "One-parameter family of factor type ratio estimators," Metron, vol. 45, no. 1-2, pp. 273-283, 1987.

[24] V. D. Naik and P. C. Gupta, "A general class of estimators for estimating population mean using auxiliary information," Metrika, vol. 38, no. 1, pp. 11-17, 1991. 
[25] S. Bahl and R. K. Tuteja, "Ratio and product type exponential estimators," Journal of Information $\mathcal{E}$ Optimization Sciences, vol. 12, no. 1, pp. 159-164, 1991.

[26] S. Mohanty and J. Sahoo, "A note on improving the ratio method of estimation through linear transformation using certain known population parameters," Sankhya, vol. 57, no. 1, pp. 93-102, 1995.

[27] L. N. Upadhyaya and H. P. Singh, "Use of transformed auxiliary variable in estimating the finite population mean," Biometrical Journal, vol. 41, no. 5, pp. 627-636, 1999.

[28] H. P. Singh and R. Tailor, "Use of known correlation coefficient in estimating the finite population mean," Statist Transaction, vol. 6, no. 4, pp. 555-560, 2003.

[29] C. Kadilar and H. Cingi, "An improvement in estimating the population mean by using the correlation coefficient," Hacettepe Journal of Mathematics and Statistics, vol. 35, no. 1, pp. 103-109, 2006.

[30] H. P. Singh, R. Tailor, and R. Tailor, "On ratio and product methods with certain known population parameters of auxiliary variable in sample surveys," Statistics and Operations Research Transactions, vol. 34, no. 2, pp. 157-180, 2010.

[31] C. Kadilar and H. Cingi, "A study on the chain ratio-type estimator," Hacettepe Journal of Mathematics and Statistics, vol. 32, pp. 105-108, 2003.

[32] S. Gupta and J. Shabbir, "Variance estimation in simple random sampling using auxiliary information," Hacettepe Journal of Mathematics and Statistics, vol. 37, no. 1, pp. 57-67, 2008.

[33] C. Kadilar and H. Cingi, "Ratio estimators in stratified random sampling," Biometrical Journal, vol. 45, no. 2, pp. 218-225, 2003. 


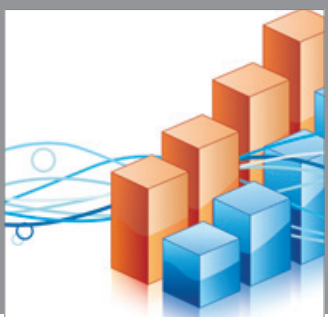

Advances in

Operations Research

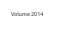

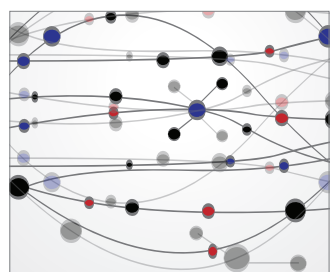

\section{The Scientific} World Journal
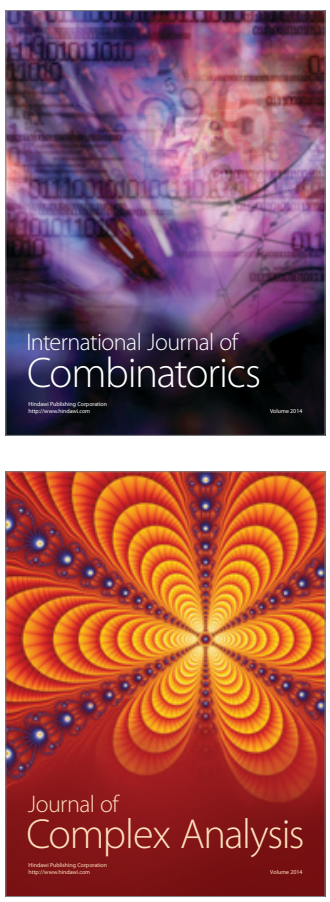

International Journal of

Mathematics and

Mathematical

Sciences
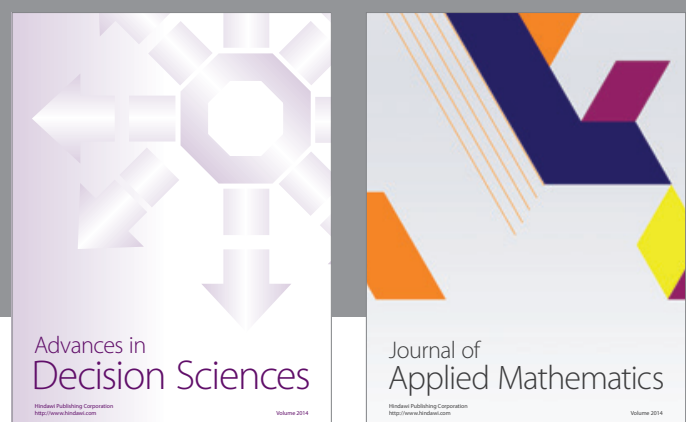

Journal of

Applied Mathematics
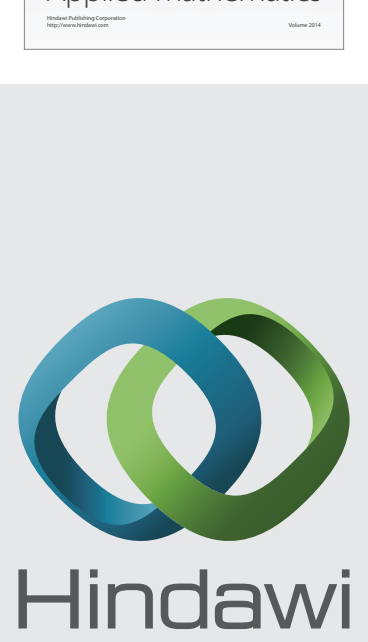

Submit your manuscripts at http://www.hindawi.com
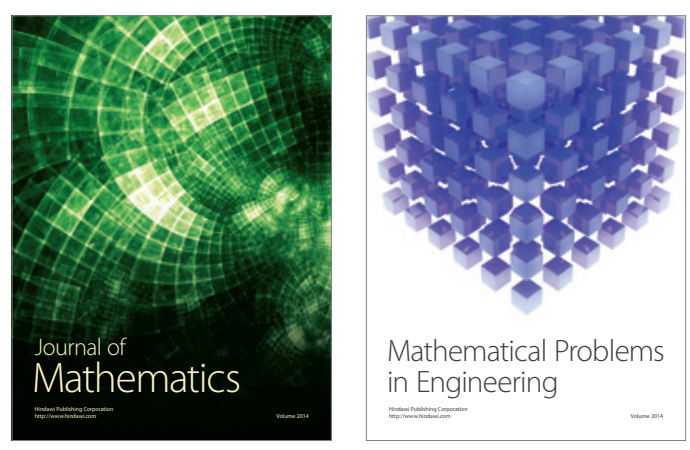

Mathematical Problems in Engineering
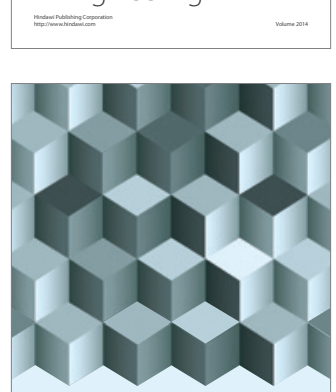

Journal of

Function Spaces
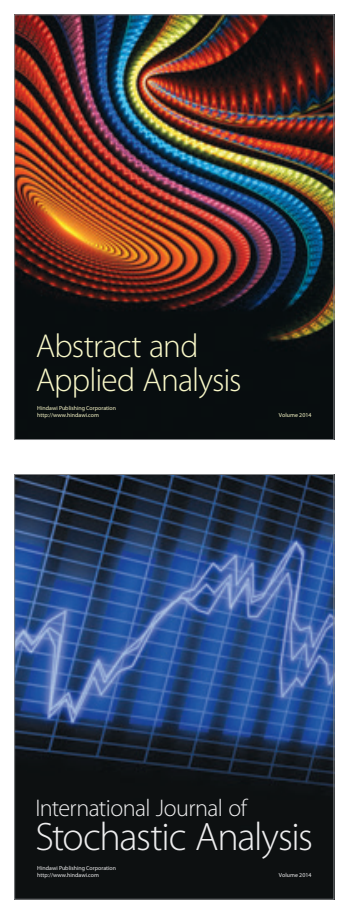

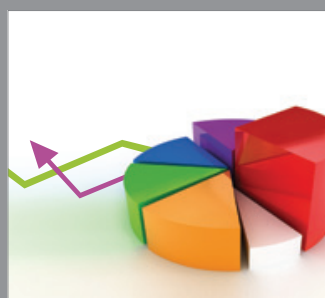

ournal of

Probability and Statistics

Promensencen
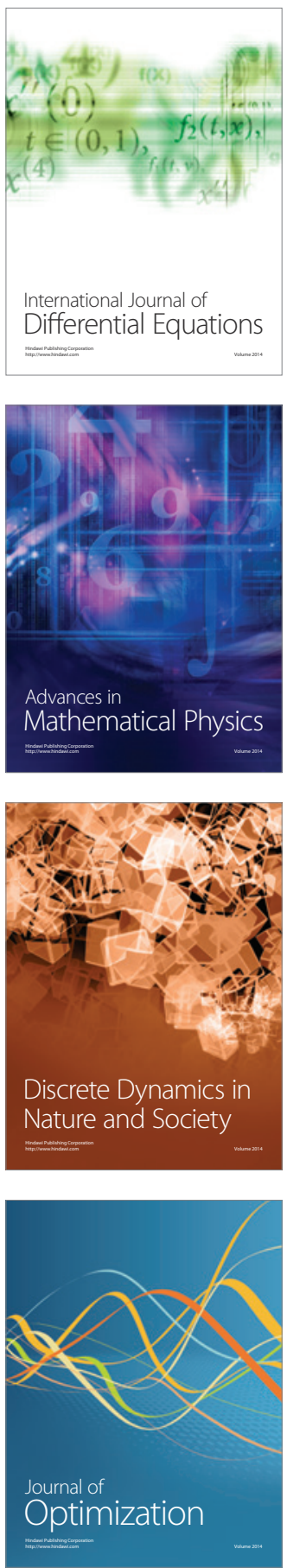